\title{
Profile of ustekinumab and its potential in the treatment of active psoriatic arthritis
}

This article was published in the following Dove Press journal:

Open Access Rheumatology: Research and Reviews

20 February 2014

Number of times this article has been viewed

\author{
Monica Montepaone' \\ Ennio Lubrano ${ }^{2}$ \\ Alessia Carboni' \\ Antonio Spadaro' \\ 'Unità Operativa Complessa di \\ Reumatologia, Dipartimento di \\ Medicina Interna e Specialità Mediche, \\ Sapienza Università di Roma, Rome, \\ ${ }^{2}$ Academic Rheumatology Unit, \\ Department of Medicine and Health \\ Sciences, University of Molise, \\ Campobasso, Italy
}

Correspondence: Antonio Spadaro Dipartimento di Medicina Interna e Specialità Mediche, UOC di

Reumatologia, Sapienza Università di Roma, Azienda Policlinico Umberto I, Viale del Policlinico 155, 0016 I Rome, Italy

Tel +390649974642

Fax +390649974642

Email a.spadaro.reuma@virgilio.it

\begin{abstract}
Psoriatic arthritis (PsA) is a chronic inflammatory arthritis and considered to be a less severe condition than rheumatoid arthritis. PsA patients have been treated for a long time with a number of different agents, from non-steroidal anti-inflammatory drugs to one or more disease-modifying antirheumatic drugs. In the last decade, recognition of the central role of tumor necrosis factor-alpha $(\mathrm{TNF} \alpha)$ in the immunopathogenesis of many rheumatic diseases, including PsA, has led to the development of TNF $\alpha$ blockers. In PsA, these agents are uniquely efficacious in the treatment of different patterns of the disease, as well as slowing progression of erosive damage in the peripheral joints. However, a significant number of patients withdraw from therapy because of failure or poor tolerability. Among the novel therapeutic targets, interleukin (IL)-23/IL-12 has been investigated for the treatment of chronic inflammatory disease. In particular, ustekinumab is a human monoclonal antibody that prevents human IL-12 and IL-23 from binding to the IL-12R $\beta 1$ receptor chain of IL-12 (IL-12R $\beta 1 / \beta 2$ ) and IL-23 (IL-12R $\beta 1 / 23 R$ ) receptor complexes on the surface of natural killer cells and T-cells. Ustekinumab has been approved only for treatment of chronic plaque psoriasis, but also represents an interesting agent for treatment of PsA.
\end{abstract}

Keywords: ustekinumab, psoriatic arthritis, psoriasis, interleukin-12, interleukin-23

\section{Introduction}

Psoriatic arthritis (PsA) is a chronic inflammatory disease typically characterized by cutaneous (ie, skin and nail disease) and articular/periarticular (peripheral arthritis, axial disease, dactylitis, enthesitis) involvement. ${ }^{1}$ Imaging techniques to assess PsA show a combination of destructive changes (joint erosions, tuft resorption, osteolysis) and bone proliferation (periarticular and shaft periostitis, ankylosis, spur formation, nonmarginal syndesmophytes $)^{2}$ with potentially progressive course, requiring an optimal management strategy. ${ }^{3,4}$ PsA is considered to be a less severe form of arthritis than rheumatoid arthritis, and has been treated for a long time with a number of different agents, from nonsteroidal anti-inflammatory drugs to one or more disease-modifying antirheumatic drugs (DMARDs) to control inflammation and/or prevent damage. The recommended nonbiological DMARDs in PsA are methotrexate (evidence level B), sulfasalazine (evidence level A), leflunomide (evidence level A), and cyclosporine (evidence level B). ${ }^{5}$ Methotrexate in particular might be considered the nonbiological DMARD of choice for the treatment of PsA, because when the cumulative probabilities of taking the different DMARDs in PsA were analyzed, methotrexate had the best survival rate. ${ }^{6}$ In addition, an observational retrospective study showed that, in real-world clinical practice, methotrexate performed well over 3 years in a group 
of patients with peripheral PsA. ${ }^{7}$ Further, a longitudinal, observational, multicenter trial studying methotrexate-naïve PsA patients in the Norwegian PsA registry showed that the 2 -year retention rate of methotrexate was $65 \%{ }^{8}$ In the last decade, recognition of the central role of tumor necrosis factor-alpha (TNF $\alpha)$ in the immunopathogenesis of many rheumatic diseases, including PsA, has led to the development of TNF $\alpha$ blockers. In PsA, these agents (adalimumab, etanercept, golimumab, infliximab) are uniquely useful in the treatment of different patterns of the disease (ie, skin and nail disease, peripheral arthritis, axial disease, dactylitis, enthesitis) as well as for slowing progressive erosive damage in the peripheral joints. Anti-TNF $\alpha$ agents in particular have been tested as monotherapy or in combination with DMARDs in randomized controlled trials and in longitudinal observational studies, and have demonstrated efficacy and safety in PsA, ${ }^{9-13}$ including the subset of patients with axial ${ }^{14}$ and early phase disease. ${ }^{15}$ Indirect analyses of placebo-controlled trials have demonstrated no significant difference between the biological agents in terms of efficacy or risk of serious adverse events. ${ }^{11,12}$ Nevertheless, it has been suggested that PsA patients with extra-articular manifestations, such as uveitis and/or inflammatory bowel disease, should be treated with monoclonal antibodies, while patients at risk of tuberculosis should be treated with etanercept. ${ }^{9}$

However, a significant proportion of patients withdraw from therapy because of failure or poor tolerability. TNF $\alpha$ antagonists have been demonstrated to be effective in PsA, with a clinical response rate ranging from $62 \%$ to $87 \%$ by Psoriatic Arthritis Response Criteria. ${ }^{16}$ The Spanish registry reported similar results $(87 \%)$ after the first year of TNF $\alpha$ treatment. ${ }^{17}$ In patients who do not respond to TNF $\alpha$ blockers, an option is to switch to another TNF $\alpha$ drug. This choice seems to be rational, owing to the different molecular structures, targets, and clinical data for the available anti-TNF $\alpha$ agents. ${ }^{17-19}$ In PsA patients, drug survival of second TNF $\alpha$ blockers after one-year was reported to be 0.81 (95\% confidence interval 0.65-0.90). ${ }^{17}$ In another study, PsA patients, who switched because of inefficacy, responded to a second-line or third-line agent in a relevant percentage of cases. ${ }^{18}$ Moreover, analysis of the pharmacoeconomic impact of TNF $\alpha$ blockers showed that this treatment was cost-effective in PsA patients with an inadequate response to traditional DMARDs. ${ }^{20}$ In the last few years, the management of PsA patients with TNF $\alpha$ blockers has become the focus for evidence-based recommendations at both the international and national level..$^{7,9,21,22}$ In particular, the Group for Research and Assessment of Psoriasis and Psoriatic Arthritis, ${ }^{21}$ the European League Against Rheumatism, ${ }^{22}$ and the Italian Society for Rheumatology have put forward recommendations for all pharmacological therapies, reporting that new biological agents different from TNF $\alpha$ blockers will emerge over the next few years. In fact, emerging evidence concerning the immunopathogenesis of PsA suggests a role for other therapeutic targets, including interleukin (IL)-1 and IL-6 blockade, B-cell depletion, inhibition of costimulation, and inhibition of receptor activator of nuclear factor- $\kappa \mathrm{B}$ (RANK)/receptor activator of nuclear factor- $\kappa \mathrm{B}$ ligand (RANKL). Among the novel targets, the IL-23/IL-17 has been investigated as a pathway for the treatment of chronic inflammatory disease. ${ }^{23,24}$

\section{IL-23/IL-I 7 pathway}

Strong association and causality between the T-cell helper (Th) 1 inductor cytokine, IL-12, and numerous immunemediated diseases was reported during the mid 1990s and led to the development of therapeutic agents targeting IL-12 function. ${ }^{25}$ IL-12 was first characterized as a product of Epstein-Barr virus-transformed human B-cell lines. ${ }^{26}$ IL-12 is a heterodimeric protein formed by two disulfidelinked, glycosylated subunits, ie, a 35 kDa light chain (p35 or IL $12 \alpha$ ) and a $40 \mathrm{kDa}$ heavy chain (p40 or IL12 $\beta$ ), and is secreted by antigen-presenting cells in response to microbial stimulation. The IL-12 receptor (IL-12R) is a heterodimeric complex consisting of IL-12R $\beta 1$ and IL-12R $\beta 2$ chains expressed on the surface of activated T-cells and natural killer cells. ${ }^{27}$ The IL-12R $\beta 1$ chain binds to the IL-12p40 subunit, whereas IL-12p35 in association with IL-12R $\beta 2$ confers an intracellular signaling ability. ${ }^{25}$ Signal transduction through IL-12R induces phosphorylation of Janus kinase (Jak2) and tyrosine kinase (Tyk2), that phosphorylate and activate signal transducer and activator of transcription (STAT)1, STAT3, STAT4, and STAT5. The specific cellular effects of IL-12 are due mainly to activation of STAT4. IL-12 induces natural killer and T-cells to produce cytokines, in particular interferon (IFN) $\gamma$, that mediate many of the proinflammatory activities of IL12, including CD4+ T-cell differentiation toward the Th1 phenotype. ${ }^{27}$

IL-23 is a member of the IL-12 cytokine family, is a heterodimer formed by a subunit or IL-23p19 (molecular weight $19,000 \mathrm{kDa}$ ), and is disulfide-linked to an additional distinct b subunit or IL-12p40 (molecular weight $40,000 \mathrm{kDa}$ ). ${ }^{28,29}$ Human p19 displays 70\% structural homology with mouse p19, and shows homology with the p35 subunit of IL-12, IL-6, and granulocyte colony-stimulating factor. ${ }^{30}$ IL-23 is mainly secreted by activated macrophages and dendritic cells in peripheral tissue (skin, intestinal mucosa, lung) 
suggesting an important role in driving the early local immune response. ${ }^{31}$ IL-23 binds to the IL-23 receptor and IL-12R $\beta 1$, but not to IL-12R $\beta 2$. The IL-23 receptor is expressed mostly on activated memory T-cells, natural killer cells, monocytes/macrophages, and dendritic cells, whereas the IL-12R $\beta 1$ chain is expressed on T-cells, natural killer cells, and dendritic cells. ${ }^{32}$

IL-23 uses Jak2 and Tyk2 to phosphorylate and activate STAT3 and STAT4. Phosphorylation of STAT4 is crucial for production of IFN $\gamma$ and subsequent differentiation of Th1 cells, whereas phosphorylation of STAT3 is essential for the development of Th17 cells. Proliferation of Th17 cells, amplified and stabilized by IL-23, results in production of IL-17. The ability of IL-23 to produce IL-17, a proinflammatory cytokine, is unique in the development and maintenance of autoimmune inflammation, compared with IL-12. ${ }^{29}$

IL-17a is a primary cytokine synthesized by Th17 cells, and has a biological role in innate and adaptive immunity. In fact, IL17a induces macrophages to produce IL-1 and TNF $\alpha$ and synovial fibroblasts secrete IL-6 and IL-8; moreover, IL17a promotes upregulation of RANKL, an inducer of osteoclastogenesis, and depletion of metalloproteinases and proteoglycans, promoting degradation of cartilage. ${ }^{23,33}$ Amplified synthesis of IL-17 has been described in patients with PsA. Activity of Th17 cells is strongly correlated with disease activity and systemic inflammation, in both early and established disease, suggesting a role of Th17 cells in both phases. ${ }^{23}$

The discovery of the IL-23/IL-17 axis has largely dispelled the paradigm that PsA is a Th1-mediated disease. ${ }^{24}$ Recognition of the fundamental role of the IL-23/IL-17 axis in the immunopathogenesis of PsA has led to the development of new agents, eg, ustekinumab, ${ }^{24,34}$ that target IL-17a signaling directly or indirectly by blocking IL-23.

\section{Ustekinumab in the treatment of PsA}

Ustekinumab is a human IgG1 kappa $(\kappa)$ monoclonal antibody against the IL-12/IL-23 p40 subunit that prevents its interaction with IL-12R $\beta 1$, thereby blocking subsequent signaling. ${ }^{29}$ Ustekinumab was generated using human Ig transgenic mice with four distinct genetic modifications that replaced the mouse Ig loci with human antibody transgenes. ${ }^{35,36}$ In vitro, ustekinumab blocks responses mediated by IL-12 and IL-23 equally. ${ }^{25}$ Ustekinumab has been investigated in four indications, ie, psoriasis, psoriatic arthritis, Crohn's disease, and multiple sclerosis, but the drug has been approved only for chronic plaque psoriasis in 74 countries all over the world, and for PsA in the US, Europe, and Japan.
Clinical evidence for the use of ustekinumab in psoriasis comes from the pivotal Phase III PHOENIX 1, PHOENIX 2, and ACCEPT studies. ${ }^{37-39}$ In PHOENIX 1, 766 patients in the US, Canada, and Belgium were randomized to receive ustekinumab or placebo for moderate-to-severe psoriasis. In this study, 255 patients received ustekinumab $45 \mathrm{mg}$ at weeks 0 and 4 and then every 12 weeks, 256 patients received ustekinumab $90 \mathrm{mg}$ in the same weeks, and 255 patients received placebo at weeks 0 and 4 , with subsequent crossover to ustekinumab at week 12 . The primary endpoint was the proportion of patients who achieved a $\geq 75 \%$ improvement in baseline Psoriasis Activity Score Index (PASI) at week 12. After two doses of ustekinumab, $67 \%$ and $66 \%$ of patients treated with $45 \mathrm{mg}$ and $90 \mathrm{mg}$, respectively, achieved PASI-75 compared with $3 \%$ of placebo-treated patients. A total of $42 \%$ and $37 \%$ of patients in the ustekinumab $45 \mathrm{mg}$ and $90 \mathrm{mg}$ groups achieved PASI-90 or nearly complete clearance of psoriasis, respectively, compared with $2 \%$ of patients in the placebo group. Of those patients that continued on ustekinumab after week $40,87 \%$ and $90 \%$ of subjects in the $45 \mathrm{mg}$ and $90 \mathrm{mg}$ groups, respectively, had a sustained PASI-75 response, compared with $64 \%$ and $62 \%$ of patients who switched to placebo. Further, $66 \%$ and $73 \%$ of patients achieved PASI-90 after $45 \mathrm{mg}$ and $90 \mathrm{mg}$ of ustekinumab, respectively, and response rates were maintained until week 52 with continued treatment. ${ }^{37}$

In PHOENIX 2, which was conducted in the US and Canada, 1,230 patients with moderate to-severe plaque psoriasis were randomly assigned to receive subcutaneous ustekinumab $45 \mathrm{mg}(\mathrm{n}=409)$, ustekinumab $90 \mathrm{mg}(\mathrm{n}=411)$, or placebo $(n=410)$ at weeks 0 and 4 , and then every 12 weeks until week 52. The primary endpoint was the proportion of patients who achieved a $\geq 75 \%$ improvement in PASI at week 12 . At week $12,67 \%$, and $76 \%$ of patients in the $45 \mathrm{mg}$ and $90 \mathrm{mg}$ arms achieved a PASI-75 response, respectively, compared with $4 \%$ of placebo-treated patients. In the same week, $42 \%$ and $51 \%$ of patients in the $45 \mathrm{mg}$ and $90 \mathrm{mg}$ groups achieved a PASI-90 versus $1 \%$ of placebo-treated patients. A number of patients who received an additional dose at week 16 maintained a response through to week 28 . Moreover, significant improvements in quality of life measures were seen within 4 weeks of treatment in ustekinumabtreated patients compared with placebo patients. Dermatology Life Quality Index scores of 6.0 were observed in both ustekinumab dosage groups, compared with a score of 1.0 in the placebo group. At week $12,72 \%$ and $77 \%$ of $45 \mathrm{mg}$-treated and $90 \mathrm{mg}$-treated patients had a significant reduction in their Dermatology Life Quality Index score, compared with 21\% 
of placebo-treated patients. A total of $49 \%, 53 \%$, and $48 \%$ of placebo-treated, $45 \mathrm{mg}$-treated, and $90 \mathrm{mg}$-treated patients, respectively, experienced adverse events. In general, these were mild and did not require adjustment of treatment. In all, $2 \%$ and $1 \%$ of $45 \mathrm{mg}$-treated and $90 \mathrm{mg}$-treated patients experienced at least one serious adverse event, compared with $2 \%$ of placebo-treated patients. ${ }^{29,38}$

ACCEPT was a randomized, 12-week, Phase III clinical trial comparing ustekinumab with etanercept. In this study, 903 subjects with moderate-to-severe psoriasis were randomized to receive ustekinumab $45 \mathrm{mg}$ or ustekinumab $90 \mathrm{mg}$ at weeks 0 and 4, or etanercept $50 \mathrm{mg}$ twice weekly. The primary endpoint was PASI-75 at week 12, which was achieved by $74 \%$ of the ustekinumab $90 \mathrm{mg}$ arm, $68 \%$ of the ustekinumab $45 \mathrm{mg}$ arm, and $57 \%$ of the etanercept arm. Ustekinumab (45 mg or $90 \mathrm{mg}$ ) was superior to etanercept, and $90 \mathrm{mg}$ of ustekinumab was significantly more efficacious than etanercept $(P<0.001)$. Further, ustekinumab at both doses resulted in a better physician global assessment than in the etanercept group. ${ }^{39}$

Clinical studies on ustekinumab in PsA included a randomized, double-blind, placebo-controlled, crossover trial $^{40}$ and the Phase III, multicenter, double-blind, placebocontrolled PSUMMIT 1 trial.

The first study ${ }^{40}$ included 24 sites in North America and Europe, and investigated 146 patients with active PsA. Using a crossover study design, the investigators randomly assigned the patients to receive subcutaneous injections of either ustekinumab $90 \mathrm{mg}$ every week for 4 weeks (weeks 0-3; total dose $360 \mathrm{mg}$ ) followed by placebo at weeks 12 and 16 (group 1, 76 patients) or placebo (weeks 0-3) followed by ustekinumab $90 \mathrm{mg}$ at weeks 12 and 16 (total dose $180 \mathrm{mg}$, group 2, 70 patients). Of these, 31 (21\%) had terminated study participation and $24(16 \%)$ had discontinued the study drug by week 36. Masking was maintained up to the infusion at week 16, and patients were followed up to week 36 . The primary endpoint was American College of Rheumatology (ACR) 20 response at week 12. At this time point, 32 (42\%) patients in group 1 and ten (14\%) in group 2 had achieved the primary endpoint ( $P=0.0002)$. Peak ACR20, ACR50, and ACR70 responses were found at weeks 16-20 in group 1 patients, and decreased slowly to week 36 . Group 2 patients had ACR20 responses similar to those in group 1 at weeks 24 (51\%, 28/55), 28 (45\%, 24/53), and 36 (42\%, 21/50).

Groups 1 and 2 were significantly different with regard to percent improvement from baseline to week 12 in tender joint count $(P<0.0001)$, patient assessment of pain $(P=0.0002)$, global assessment of disease activity by patient and doctor
$(P<0.0001)$, and Health Assessment Questionnaire (HAQ) disability index $(P=0.0075)$. At week 12, 59\% (43/73) of group 1 patients and 30\% (19/63) group 2 patients achieved a good-to-moderate DAS28 rating $(P=0.0009)$. At week 24, when patients in group 2 had crossed over from placebo to receive two doses of ustekinumab, $60 \%(33 / 55)$ of patients achieved a good-to-moderate DAS28 rating.

At week 12 , there was a significantly $(P=0.0163)$ lower proportion of patients with enthesopathy in group $1(23 \%)$ compared with group 2 (42\%). Similar proportions of group 1 patients (38\%) and group $2(33 \%)$ patients had dactylitis at week $12(P=0.5429)$. However, at week 12, patients with dactylitis at baseline showed improved median scores in group 1 versus group 2 (2.0 versus $0.0 ; P=0.0107)$. By week 12, adverse events were found in $90(62 \%)$ patients, and discontinuation was necessary in five cases (3\%). Infectious complications occurred in a third of patients up to week 12 (36\% of group 1 and $30 \%$ of group 2). No serious adverse events occurred in group 1 patients up to week 12, whereas three were recorded in group 2 (myocardial infarction in a 35-year-old woman, non-cardiac chest pain, and a hemorrhagic gastric ulcer). Another serious adverse event was reported after week 12 by a group 2 patient (a 40 -year-old woman with a hemorrhagic abdominal cyst) who did not receive ustekinumab at weeks 12 and 16. The pattern of adverse events at week 36 was similar to that seen during the initial placebo-controlled, 12-week period. Six additional ustekinumab-treated patients had serious adverse events. A basal cell cancer was reported in an individual who had had two previous basal cell cancers. No deaths, cases of tuberculosis, or opportunistic infections occurred during the study. Over the 36 weeks, 11\% (14/124) of patients developed antibodies to ustekinumab, most of which were low titer, but injection site reactions did not appear. ${ }^{40}$

More recently, a multicenter, double-bind, placebocontrolled Phase III trial (PSUMMIT1) was undertaken at 104 sites in 14 countries, including North America, Europe, Australia, and New Zealand. ${ }^{41}$ The study population comprised 615 adults with active PsA. Although 319 (51.9\%) patients were not taking methotrexate in association, most (roughly 75\%) had received methotrexate previously. Patients were randomly assigned to $45 \mathrm{mg}$ or $90 \mathrm{mg}$ of ustekinumab or placebo at week 0,4 , and every 12 weeks thereafter.

At week 16, patients receiving placebo with less than $5 \%$ improvement in both tender and swollen joint counts switched to ustekinumab $45 \mathrm{mg}$, while patients receiving ustekinumab $45 \mathrm{mg}$ with less than 5\% improvement in joint counts were given ustekinumab $90 \mathrm{mg}$. Patients receiving 
ustekinumab $90 \mathrm{mg}$ continued their masked dose regimen. Patients taking placebo who did not escape early, crossed over to receive ustekinumab $45 \mathrm{mg}$ at week 24 , week 28 , and every 12 weeks thereafter. Ustekinumab-treated patients received placebo injections at week 20 and week 24 to maintain masking. ${ }^{41}$ The primary endpoint of PSUMMIT1 was the proportion of patients with at least an ACR20 response at week 24. A significantly higher proportion of patients in the ustekinumab groups than in the placebo group achieved an ACR20 response at week $24(P<0.0001)$. ACR20 responses in the ustekinumab groups differed significantly from those in the placebo group by week $8(P<0.0001)$ and improved with time. The highest ACR20 response rates occurred at week 28, and were maintained at week 52. Significant differences were found at week 24 for ACR50 response (26.4\% in the combined ustekinumab group; $24.9 \%$ for ustekinumab $45 \mathrm{mg} ; 27.9 \%$ for ustekinumab $90 \mathrm{mg}$; and $8.7 \%$ for placebo; $P<0.0001$ for all placebo comparisons) and for ACR70 response (13.2\% for the combined ustekinumab group; $12.2 \%$ for ustekinumab $45 \mathrm{mg} ; 14.2 \%$ for $90 \mathrm{mg}$ ustekinumab; and $2.4 \%$ for placebo; $P<0.0001$ for all placebo comparisons). The proportion of patients achieving an ACR50 response (38\% of patients on placebo switched to the $45 \mathrm{mg}$ group and $34.2 \%$ to the combined ustekinumab group) and an ACR70 response ( $16.3 \%$ on placebo switched to the $45 \mathrm{mg}$ group and $19.6 \%$ to the combined ustekinumab group) improved further from week 24 to week 52 . Of the patients with dactylitis at baseline, significantly lower proportions in the ustekinumab treatment groups $(56.2 \%$ in the combined group, $56.6 \%$ in the ustekinumab $45 \mathrm{mg}$ group, and $55.8 \%$ in the ustekinumab $90 \mathrm{mg}$ group) had digits with dactylitis at week 24 ( $P=0.0013$, $P=0.0050$, and $P=0.0038$, respectively) compared with the placebo group (76.1\%). Compared with placebo, the ustekinumab groups showed significantly greater improvements in dactylitis scores $(P=0.0003)$ and enthesitis scores $(P=0.0019$ for the $45 \mathrm{mg}$ group and $P<0.0001$ for the $90 \mathrm{mg}$ group). Of the patients with spondylitis at baseline, a significantly $(P<0.0137)$ greater proportion in the ustekinumab $90 \mathrm{mg}$ group achieved responses on the Bath Ankylosing Spondylitis Disease Activity Index 50 compared with those on placebo. In patients with at least $3 \%$ of body surface area with psoriasis at baseline, at week 24, significantly greater proportions of patients in the ustekinumab groups than in the placebo group achieved PASI-75 $(P<0.0001)$ or at least a $90 \%$ improvement in baseline scores on the index ( $42.5 \%$ patients in the ustekinumab groups versus $2.7 \%$ in the placebo group, $P<0.0001$ ). At week 52, 69.1\% of ustekinumab-treated patients achieved PASI-75 and 49.5\% achieved PASI-90. Even improvements in HAQ Disability Index scores at week 24 were significantly greater in patients given ustekinumab compared with those on placebo $(P<0.0001$ for all comparisons). Significantly more ustekinumab-treated patients achieved a clinically meaningful improvement of 0.3 or more in HAQ Disability Index scores than in the placebo group (47.7\% versus $28.2 \%$, respectively, $P<0.0001)$. Improvements in physical function were maintained at week 52 .

At week 16, the appearance of adverse events was similar in the ustekinumab and placebo groups (41.8\% versus $42 \%$, respectively); similar rates of infections and serious adverse events were also described. The most common adverse events in ustekinumab-treated patients were nasopharyngitis (4.6\% of patients), upper respiratory tract infections $(3.4 \%)$ and headache $(3.4 \%)$. The proportions of patients reporting adverse events and the types of adverse events did not seem to differ according to whether or not methotrexate was being administered concomitantly. Adverse events increased in frequency at week 52, but this did not seem to be dose-related. No opportunistic infections (including tuberculosis), death, or malignancies were noted by week 52 . After week 24 , cholecystitis was noted in two patients (one in the placebo group who escaped early to $45 \mathrm{mg}$ ustekinumab, and one in the $45 \mathrm{mg}$ group), salpingitis in one patient in the $45 \mathrm{mg}$ group, erysipelas in one patient in the $90 \mathrm{mg}$ group, and a pharyngolaryngeal abscess in one patient in the $90 \mathrm{mg}$ group. No major adverse cardiovascular events appeared by week 16. Between week 16 and week 24, a nonfatal stroke was reported in a 53-year-old former smoker with pre-existing hypertension and hyperlipidemia who had had a previous cerebrovascular event necessitating internal carotid artery stenting. This patient was initially assigned to ustekinumab $45 \mathrm{mg}$ and did not escape early. Between week 24 and week 52, two additional patients (who were both originally given placebo and escaped early to $45 \mathrm{mg}$ ustekinumab) had myocardial infarctions. By week 52, injection site reaction was noted in four (1\%) patients in the ustekinumab $45 \mathrm{mg}$ group, five $(2.1 \%)$ in the ustekinumab $90 \mathrm{mg}$ group, and ten $(1.6 \%)$ in the placebo group. Injection site reactions did not require discontinuation of the study drug. By week 52, no associated anaphylactic or serum sickness-like reactions were noted. ${ }^{41}$

\section{Conclusion}

Beyond TNF $\alpha$ blockade, the relevant immunopathogenetic role of the IL-23/IL-17 axis has modified the paradigm that PsA is a Th1-mediated disease and stimulated the development of new drugs that interfere with this pathway. 
Thus, new biological agents with other mechanisms of action might prove to be the optimal approach for treatment of this intriguing disease. Ustekinumab represents a valid approach beyond anti-TNF $\alpha$. Whether ustekinumab is able to halt the progression of the disease is still debatable.

\section{Disclosure}

The authors report no conflicts of interest in this work.

\section{References}

1. Kavanaugh AF, Ritchlin CT. GRAPPA Treatment Guideline Committee. Systematic review of treatments for psoriatic arthritis: an evidence based approach and basis for treatment guidelines. J Rheumatol. 2006;33:1417-1421.

2. Spadaro A, Lubrano E. Psoriatic arthritis: imaging techniques. Reumatismo. 2012;64(2):99-106.

3. McHugh NJ, Balachrishnan C, Jones SM. Progression of peripheral joint disease in psoriatic arthritis: a 5-yr prospective study. Rheumatology (Oxford). 2003;42:778-783.

4. Lubrano E, Perrotta FM, Carboni A, Scarno A, Ferrara N, Spadaro A. Disease-modifying antirheumatic drugs or biological blockers in psoriatic arthritis? CML Rheumatology. 2013;32:77-83.

5. Soriano ER, McHugh NJ. Therapies for peripheral joint disease in psoriatic arthritis. A systematic review. $J$ Rheumatol. 2006;33:1422-1430.

6. Spadaro A, Taccari E, Mohtadi B, Riccieri V, Sensi F, Zoppini A. Life-table analysis of cyclosporin A treatment in psoriatic arthritis: comparison with other disease-modifying antirheumatic drugs. Clin Exp Rheumatol. 1997;15:609-614.

7. Ricci M, De Marco G, Desiati F, et al. Long term survival of methotrexate in psoriatic arthritis. Reumatismo. 2009;61:125-131.

8. Lie E, van der Heijde D, Uhlig T, et al. Effectiveness and retention rates of methotrexate in psoriatic arthritis in comparison with methotrexate-treated patients with rheumatoid arthritis. Ann Rheum Dis. 2010;69:671-676.

9. Salvarani C, Pipitone N, Marchesoni A, et al; Italian Society for Rheumatology. Recommendations for the use of biologic therapy in the treatment of psoriatic arthritis: update from the Italian Society for Rheumatology. Clin Exp Rheumatol. 2011;29:28-41.

10. Spadaro A, Ceccarelli F, Scrivo R, Valesini G. Life-table analysis of etanercept with or without methotrexate in patients with psoriatic arthritis. Ann Rheum Dis. 2008;67:1650-1651.

11. Saad AA, Symmons DP, Noyce PR, Ashcroft DM. Risks and benefits of tumor necrosis factor-alpha inhibitors in the management of psoriatic arthritis: systematic review and meta-analysis of randomized controlled trials. J Rheumatol. 2008;35:883-890.

12. Ash Z, Gaujoux-Viala C, Gossec L, et al. A systematic literature review of drug therapies for the treatment of psoriatic arthritis: current evidence and meta-analysis informing the EULAR recommendations for the management of psoriatic arthritis. Ann Rheum Dis. 2012;71:319-326.

13. Saad AA, Hyrich KL, Ashcroft DM. Drug persistence, effectiveness and safety assessment of anti-TNF therapies in psoriatic arthritis. Expert Opin Drug Saf. 2011;10:219-226.

14. Lubrano E, Spadaro A, Marchesoni A, et al. The effectiveness of a biologic agent on axial manifestations of psoriatic arthritis. A twelve months observational study in a group of patients treated with etanercept. Clin Exp Rheumatol. 2011;29:80-84.

15. Scarpa R, Atteno M, Lubrano E, et al. The effectiveness and safety of TNF-alpha blockers in the treatment of early psoriatic arthritis: an Italian multicentre longitudinal observational pilot study. Clin Rheumatol. 2011;30:1063-1067.

16. Kavanaugh A, Tutuncu Z, Catalan-Sanchez T. Update on anti-tumor necrosis factor therapy in the spondyloarthropathies including psoriatic arthritis. Curr Opin Rheumatol. 2006;18:347-353.
17. Gomez-Reino JJ, Carmona L; BIOBADASER Group. Switching TNF antagonists in patients with chronic arthritis: an observational study of 488 patients over a four-year period. Arthritis Res Ther. 2006;8(1):R29.

18. Coates LC, Cawkwell LS, Ng NW, et al. Sustained response to longterm biologics and switching in psoriatic arthritis: results from real life experience. Ann Rheum Dis. 2008;67:717-719.

19. Brocq O, Roux $\mathrm{CH}$, Albert $\mathrm{C}$, et al. TNF alpha antagonist continuation rates in 442 patients with inflammatory joint disease. Joint Bone Spine. 2007;74:148-154.

20. Olivieri I, de Portu S, Salvarani C, et al; PACE working group. The psoriatic arthritis cost evaluation study: a cost-of-illness study on tumour necrosis factor inhibitors in psoriatic arthritis patients with inadequate response to conventional therapy. Rheumatology. 2008;47:1664-1670.

21. Ritchlin CT, Kavanaugh A, Gladman DD, et al; Group for Research and Assessment of Psoriasis and Psoriatic Arthritis (GRAPPA). Treatment recommendations for psoriatic arthritis. Ann Rheum Dis. 2009;68: 1387-1394

22. Gossec L, Smolen JS, Gaujoux-Viala C, et al. European League Against Rheumatism recommendations for the management of psoriatic arthritis with pharmacological therapies. Ann Rheum Dis. 2012;71:4-12.

23. Leipe J, Grunke M, Dechant C, et al. Role of Th17 cells in human autoimmune arthritis. Arthritis Rheum. 2010;62:2876-2885.

24. Spadaro A, Lubrano E. Beyond anti-TNF- $\alpha$ agents in psoriatic arthritis. Expert Rev Clin Immunol. 2013;9:507-509.

25. Benson JM, Sachs CW, Treacy G, et al. Therapeutic targeting of the IL-12/23 pathways: generation and characterization of ustekinumab. Nat Biotechnol. 2011;29:615-624.

26. Kobayashi M, Fitz L, Ryan M, et al. Identification and purification of natural killer cell stimulatory factor (NKSF), a cytokine with multiple biologic effects on human lymphocytes. J Exp Med. 1989;170:827-845.

27. Trinchieri G. Interleukin-12 and the regulation of innate resistance and adaptive immunity. Nat Rev Immunol. 2003;3:133-146.

28. Kleinschek MA, Muller U, Brodie SJ, et al. IL-23 enhances the inflammatory cell response in Cryptococcus neoformans infection and induces a cytokine pattern distinct from IL-12. J Immunol. 2006;176: 1098-1106.

29. Tang C, Chen S, Qian H, Huang W. Interleukin-23: as a drug target for autoimmune inflammatory diseases. Immunology. 2012;135: $112-124$.

30. Oppmann B, Lesley R, Blom B, et al. Novel p19 protein engages IL-12p40 to form a cytokine, IL-23, with biological activities similar as well as distinct from IL-12. Immunity. 2000;13:715-725.

31. McKenzie BS, Kastelein RA, Cua DJ. Understanding the IL-23-IL-17 immune pathway. Trends Immunol. 2006;27:17-23.

32. Jana M, Pahan K. Induction of lymphotoxin-alpha by interleukin-12 p40 homodimer, the so-called biologically inactive molecule, but not IL-12 p70. Immunology. 2009;127:312-325.

33. Iwakura Y, Ishigame H. The IL-23/IL-17 axis in inflammation. $J$ Clin Invest. 2006;116:1218-1222.

34. Patel DD, Lee DM, Kolbinger F, Antoni C. Effect of IL-17 A blockade with secukinumab in autoimmune diseases. Ann Rheum Dis. 2013; 72 Suppl 2:S116-S123.

35. Fishwild DM, O'Donnell SL, Bengoechea T, et al. High-avidity human $\operatorname{IgG}$ (kappa)monoclonal antibodies from a novel strain of minilocus transgenic mice. Nat Biotechnol. 1996;14:845-851.

36. Lonberg N. Human antibodies from transgenic animals. Nat Biotechnol. 1995;23:1117-1125.

37. Leonardi CL, Kimball AB, Papp KA, et al; PHOENIX 1study investigators. Efficacy and safety of ustekinumab, a human interleukin12/23 monoclonal antibody, in patients with psoriasis: 76-week results from a randomised, double-blind, placebo-controlled trial (PHOENIX 1). Lancet. 2008;371:1665-1674.

38. Papp KA, Langley RG, Lebwohl M, et al; for PHOENIX 2 study investigators. Efficacy and safety of ustekinumab, a human interleukin12/23 monoclonal antibody, in patients with psoriasis: 52-week results from a randomised, double-blind, placebo-controlled trial (PHOENIX 2). Lancet. 2008;371:1675-1684. 
39. Griffiths CE, Strober BE, van de Kerkhof P, et al; ACCEPT Study Group. Comparison of ustekinumab and etanercept for moderate-to-severe psoriasis. N Engl J Med. 2010;362:118-128.

40. Gottlieb A, Menter A, Mendelsohn A, et al. Ustekinumab, a human interleukin 12/23 monoclonal antibody, for psoriatic arthritis: randomised, double-blind, placebo-controlled, crossover trial. Lancet. 2009;373:633-640.
41. McInnes IB, Kavanaugh A, Gottlieb AB, et al. PSUMMIT 1 Study Group. Efficacy and safety of ustekinumab in patients with active psoriatic arthritis: 1 year results of the phase 3, multicentre, double-blind, placebo-controlled PSUMMIT 1 trial. Lancet. 2013;382:780-789.

\section{Publish your work in this journal}

Open Access Rheumatology Research and Reviews is an international peer-reviewed, open access journal, publishing all aspects of clinica and experimental rheumatology in the clinic and laboratory including the following topics: Pathology, pathophysiology of rheumatologica diseases; Investigation, treatment and management of rheumatological diseases; Clinical trials and novel pharmacological approaches for the treatment of rheumatological disorders. The manuscript management system is completely online and includes a very quick and fair peerreview system, which is all easy to use. Visit http://www.dovepress.com/ testimonials.php to read real quotes from published authors.

\footnotetext{
Submit your manuscript here: http://www.dovepress.com/open-access-rheumatology-research-and-reviews-journal
} 